\title{
Principal component analysis for decision making in healthcare and hospital readmissions
}

Taiwo Ajani, Associate Professor Computer Technology and Information Systems, Ferrum College, VA, tajani@ferrum.edu

George Habek, Senior Manager, Analytics Solutions, CT Global Solutions, PA, ghabek@msn.com

\begin{abstract}
The authors examined datafiles from a hospital system and applied various analytical techniques including principal component analysis to determine the drivers of readmission. Results were discussed with respect to Medicare "all-cause" 30-day readmission policy. The study highlights decision support value of hospital data and the need to utilize them for continuing healthcare quality management, cost reduction and patient outcomes.
\end{abstract}

Keywords: healthcare, analytics, medicare, readmission, pca, regression

\section{Introduction}

Healthcare quality, performance and costs have led to continuing call for evaluation of readmission rates across hospital systems. Generally, hospital readmission occurs when a patient is admitted back to the hospital within a defined or specified time after an initial discharge. Medicare "all-cause" specifies hospital stays within 30 days of discharge from initial hospitalization as readmission regardless of reasons. According to Boccuti and Casillas (2015), this approach is used in calculating both individual hospital and national average readmission rates. As institutional oversights become more pronounced over hospitalization and readmission especially in the past decade, the healthcare industry also experienced unprecedented integration of Information Technology and systems. These events have led to massive creation of digital data which in turn provided opportunities for analyses. Increased knowledge of- and integration- of various analytical professionals, tools and techniques in this domain, continue to expand opportunities for healthcare quality improvement and cost reduction. This study investigates readmission datasets using various model building techniques; discusses theoretical background for readmission and provides suggestions for mitigating the effects of variables driving readmission.

\section{Theoretical Background}

Several authors have investigated inter alia hospital readmission rates programs (HRRP) with respect to specific diseases such as, heart failure (Blecker et al, 2019; Herrin et al., 2015, Blecker et al., 2013), dementia (Ma et al., 2019), cirrhosis and liver failure (Shaheen, 2019), multiple chronic conditions cluster (Basu, Avila and Ricciardi, 2016), and even surgery (Goel et al., 2019; Merkow, 2015). 


\section{Issues in Information Systems}

Volume 22, Issue 4, pp. 334-345, 2021

Herrin et al. (2015) examined the relationship between community factors and hospital readmission rates (HRR). In their observation of patients that presented with acute myocardial infarction, heart failure and pneumonia, Herrin and his coauthors reported that community factors as measured by county characteristics explain a substantial amount of variation in HRR. Several other authors dedicated a fair amount of their literature on healthcare costs and the Medicare efforts at establishing HRR program with integrated penalties to incentivize a reduction of readmission rates in hospital systems. According to Herrin et al. (2015), hospital leaders across the country focus on improving 30-day readmission rates to control costs and improve patient care. Understanding the causes for variation in HRR is very critical to improving rates. Certain researchers focus on variation at the hospital level including teaching status, staffing, bed size and ownership (Joynt, Orav, and Jha, 2011; Krumholz et al. 2009). Tsugawa et al. (2017) observed that elderly patients treated by male internists had higher mortality and readmission rates than those that were treated by female internists, a study that gender and associated differences in practice culture or pattern have important implications for health quality and patient outcomes.

Others focused at the patient-level (Merkow et al., 2015; Barnett, Hsu \& McWilliams, 2015). For instance, Barnett et al. (2015) in their study on Patient Characteristics and differences in hospital readmission rates concluded that certain patient characteristics explained a lot of the differences observed in readmission risks between patients admitted to hospitals with higher vs lower readmission rates. Such data can be used by quality auditors to penalize hospitals with higher readmission rates. Merkow et al. (2015), observed that post-discharge complications are indicators for readmission after surgery. The authors suggested that such data should be considered when healthcare quality institutions create indicators and policies for penalizing hospital systems for surgical readmission. According to Boccuti and Casillas (2015), the CMS first imposed readmission penalties in the 2013 fiscal year, of a maximum penalty $1 \%$ of the hospital's base inpatient claims. Penalties levied in 2013 and 2014 included three selected conditions: chronic obstructive pulmonary disease COPD, and elective hip or knee replacement. As CMS expanded the conditions measured, the proportion of hospitals receiving penalties increased to about $78 \%$.

This was the intent of the HRR program (HRRP) when it started. According to Sheingold, Zuckerman, and Shartzer (2013), the Centers for Medicaid and Medicare Services (CMS), as part of the HRR program, began penalizing hospitals that had excess patient readmissions within 30 days of initial discharge. The practice was instituted by the Affordable Care Act to address the seemingly intractable high rates of readmissions which reflected poorly upon quality of care, and skyrocketing costs to Medicare program. Subsequent studies showed that instituted penalties helped to reduce readmission rates (Desai et al., 2016). Hospitals on their part, often respond to the HRRP with multimodal strategies including improved patient education, post-discharge follow-up, and risk stratification among others (Blecker et al., 2019). Interventions at the hospital level and legislative policies at the governmental level benefit healthcare quality, cost reduction and patient outcomes. However, optimization of healthcare at this level depends on massive health data at different levels (including, patient, hospital, geography, clinic etc.) that may reside in several silos, requiring integration and massive cleaning for analytics studies.

Ajani and George (2019a) described several tools and techniques that are suitable for analytics in the healthcare domain. Every tools and techniques have pros and cons, including costs of acquisition and/or availability; although, benefit and time may sometimes outweigh the cost argument. Where practitioners have access to these tools, the onus is on the expert to select what can best advance analyses. In this article we demonstrate the use of analytical techniques an analytical process conducted on the readmission data of a large hospital system. 


\section{Issues in Information Systems}

Volume 22, Issue 4, pp. 334-345, 2021

\section{Typical Business Challenge}

A large hospital system in the US wants to establish drivers of hospital readmission; with the goal to enact policies that can help improve outcomes, enhance quality and lower costs through reduced readmission rates. All patients were admitted into the hospital, many were discharged, some died while several were readmitted. Each patient had two identification numbers i.e. encounter key and patient key. The readmission datafiles need to be examined at the granular visit level (encounter key) and not at the patient level (patient ID). Otherwise, the records have to be de-duplicated since one patient could have several visits. The hospital system is interested on every visit because more visits tend to suggest problems hence the use of the encounter key which defines each visit as unique. Using historical data of about 160,000 records, we seek to provide answers to the following question: What are the causes/drivers for a patient being readmitted to the hospital within 30-60 days after discharge?

\section{Methodology}

Four distinct data sources were identified for this article as follows: (a) Hospital table contained information about the patients' interaction with, and at the various hospital sites that form part of the hospital system. Hospital data was in .XLSX format and contained such variables as length_of_stay and department; (b) Demographic table contained information such as the patients' age and gender (from the insurance provider in a .CSV delimited format); (c) Geo-map table contained geographical location such as city and state in a .TAB delimited format. (d) The Condition table is made up of columns such as diagnosis, procedure etc.

\section{The Data Mining Process}

\section{Standard Data Quality Management}

Data Management and analyses was accomplished using the R Studio. Several packages and libraries were installed including lattice, ggplot2, dplyr, and Hmisc etc. Once the data files were merged into one dataframe, we explored and investigated the quality of the data and standardized for subsequent analyses. Some of the dimensions and activities include accuracy and ensuring that fields have correct values; completeness to ensure that data fields have values; missing values in numerical fields were replaced with the appropriate value of central tendency (CT). For instance, missing age values were replaced with the median value. Median value was preferred over mean because of the outliers that were observed within the age variable. We also checked values for consistency to remove contradictions from field values. We checked that values were up to date (currency) and relevant in terms of meta-data or data items with value. We confirmed that there was validity in the datasets; and data fields contained allowable values. Finally, it was important that each record is unique therefore, duplicates were removed where they existed.

\section{Data cleansing and wrangling}

The first step was to merge the four datafiles. The resulting dataframe was explored for missing data, and bad data entry. For instance, for Patient Age, zeros were replaced with missing; question marks of the type "??" were replaced with blanks in the Diagnosis column etc. Specifically, scripts were written to replace asterisks with blanks and converts the number of chronic condition column into numerics. The Department column was a little tricky because R stored it as factor. Therefore, it was first converted into character as the computation was giving dummy figures; hence the question marks "??" were replaced with blanks. Other columns were similarly treated. Since all the variables were not relevant to the objectives of the investigation, a subset of relevant columns (variables) were created. The summary() and describe() 


\section{Issues in Information Systems}

Volume 22, Issue 4, pp. 334-345, 2021

functions were used for further exploration and we observed some duplicate data. The merged data file was subsequently deduplicated.

\section{Results}

\section{Descriptive Results}

Initial exploration of the data showed about 160,000 records consisting of about $90 \%, 5 \%$ and $3.5 \%$ patients from Florida, Alabama and Georgia respectively. The states of Illinois, Missouri, Tennessee, Arkansas, Mississippi, Virginia and Texas were each represented by less than $1 \%$ of the patients. Patients' age ranged from 27 to 95 years with a mean value of 74.44 years. The data showed a mostly aging population characterized by citizens between 65 and 85 years. This explains the observed old age diseases of chronic heart failure (CHF), acute myocardial infraction (AMI), chronic obstructive pulmonary disease (COPD). Gender was approximately split into 56:44 ratio for men and women respectively. Of the total observation, 31,188 returned to the hospital after initial discharge representing about $19.5 \%$ readmission. The top three facilities that patients were discharged to include routine discharge/home (62.5\%), home health agency (18\%), and skilled nursing facility (10.9\%). The average cost of a hospital stay was about $\$ 28,800$ while the highest recorded cost was $\$ 67,671$. The lowest recorded value was negative number. This is most likely a recording error, calculation error or a refund from the hospital processes. These records would be best used for a financial report once the outliers are removed. Finally, In the case of the readmission data, since there were a large number of missing values, those missing values should be replaced by the median record of the given column data. There were not many recorded patients who spent an extensive amount of time in the hospital. Most patients spent about three days in the hospital. Likewise, a considerable number of patients had no surgeries. Very few patients spent more than 15 days in the hospital (see figure 1 below).

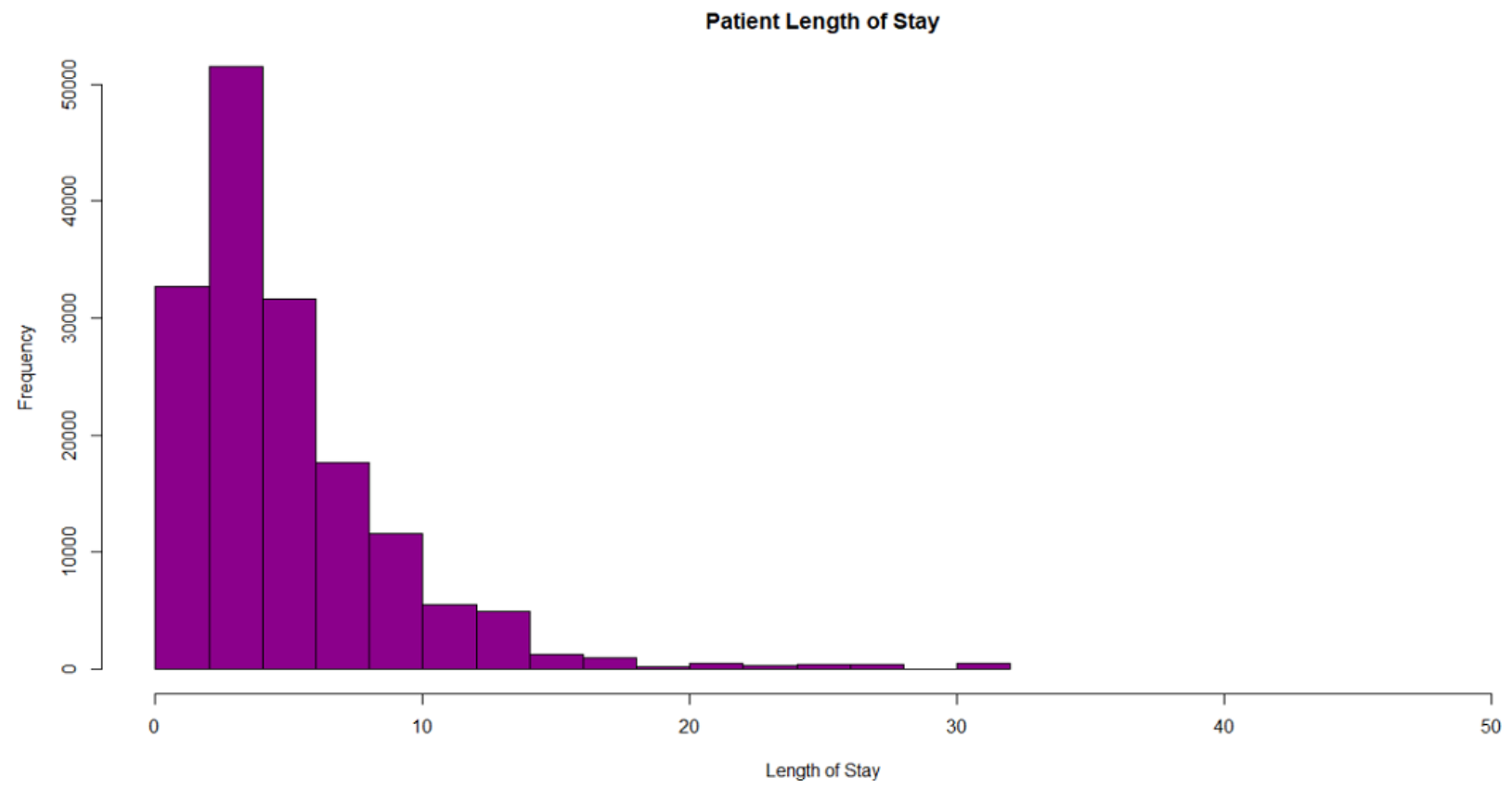

Figure 1. Patients' Length of stay 


\section{Issues in Information Systems}

Volume 22, Issue 4, pp. 334-345, 2021

There were three diagnostic groups of acute myocardial infection (AMI), congestive heart failure (CHF), chronic obstructive pulmonary disease (COPD) of ratio 27.4:65.0:7.6 respectively. Hospital visits cost these patients about 29,000 dollars on average from a range of 0 to $>67500$ dollars. The number of days patients were admitted to the ICU ranged from 0 - 29 days with $50 \%$ of the patients staying at least 2 days in the ICU. Within groups (race, diagnosis group, gender), significant discrepancies were not observed in the hospital length of stay or total service costs. Of the two-third of the population with recorded data for chronic diagnoses, about ninety percent were diagnosed with 3 chronic conditions at least. Hospital procedure that were performed on patients ranged from a minimum alcohol detoxification and arterial catheterization to transfusion of packed cell, trans-jugular liver biopsy, and electroencephalographic monitoring at the upper-end. About $59 \%$ of the total observation went through (at least) one surgical operation.

\section{Model Development}

The first step was to separate the datasets that was used to score the model which was accomplished by installing the necessary caret package and calling it in R studio. The seed was set and $10 \%$ score data was created from the readmission data. The resulting dataframe was subsequently partitioned into training and validation datasets.

\section{Principal Component Analysis}

The Principal Component Analyses (PCA) is often used for feature selection and engineering. Additionally, we used the technique to make decision on which of the features should be included in the final model chosen from the models explored. The PCA technique was selected to reduce variable dimensionality. Although the number of variables in the study data was about fifty in number and it is easy to select relevant variables from it. However, consider a situation where you have several thousand variables, automated column selection becomes imperative to reduce variable dimensionality. A number of other techniques are available including clustering. It should be noted that PCA works better with numeric columns which in this case were selected and parsed into myvarspca. In order to first test for multicollinearity, Spearman's correlation was performed. Since both the Length of stay and days_in_ICU variables seem to be modeling the same values as they were observed to linearly correlate, this was noted and was considered in our debate to select the final model. 


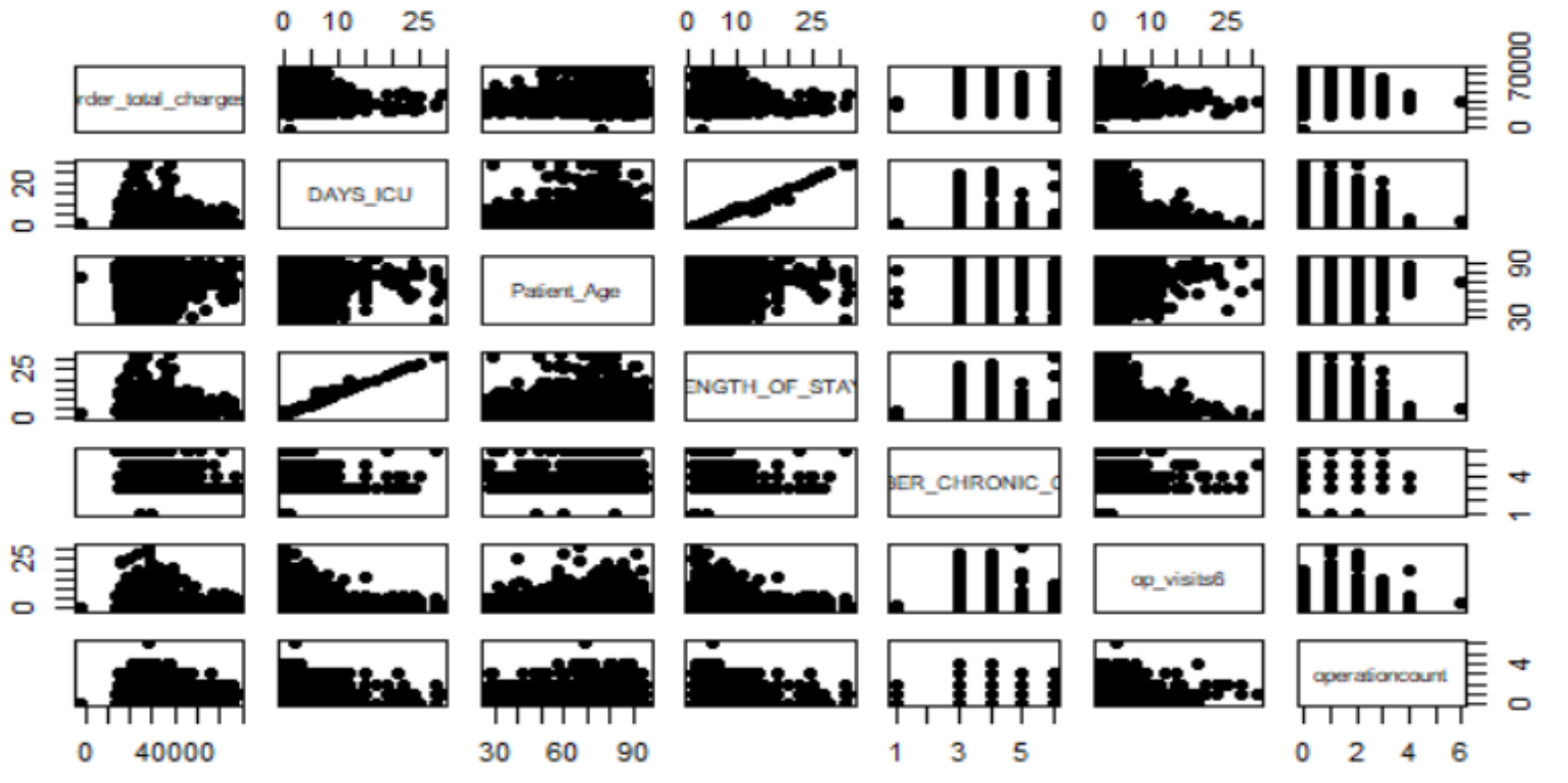

Figure 2. Correlation between variables

The PCA technique does not remove features. Instead, it creates new variables using original features. Principal Component Analysis is a feature extraction technique. The principal components are "new" variables (linear combination of original features) that are independent of one another and help to identify most important variables for predicting the response. PCA is better with numeric data types. In the assignment dataset, there a few categorical features having numeric data types, such as zip, diagnostic codes, hospital co-ordinates, order set used, admit month etc. These features will not be included in PCA. The eigenvalue and variance percentage of principal components (PC) are given below. The cumulative variance of $84 \%$ is covered by first 4 PCs (in the order of importance). This is an acceptable large percentage of variance explained by PC. The lowest value variables - PC 5 \& PC 6, can be safely discarded.

PATIENT_AGE
ADMIT_LOS
ICU_DAYS
NUM_VISITS
NUM_CHRONIC_COND
OPERATION_COUNT

$\begin{array}{rrrrr}\text { Dim.1 } & \text { Dim.2 } & \text { Dim.3 } & \text { Dim.4 } & \text { Dim.5 } \\ 0.002318466 & 6.31265180 & 0.177750798 & 92.080302645 & 1.425876865 \\ 49.901870264 & 0.06463311 & 0.003084805 & 0.009685963 & 0.021858409 \\ 49.899095088 & 0.07068049 & 0.005700978 & 0.020403014 & 0.006500639 \\ 0.031881723 & 51.81148490 & 0.400820199 & 1.127260848 & 46.626737038 \\ 0.083133409 & 9.40712828 & 69.328624762 & 2.888880240 & 18.292232808 \\ 0.081701050 & 32.33342142 & 30.084018457 & 3.873467290 & 33.626794241\end{array}$

Figure 3: Eigenvalue for Principal Component Analysis 


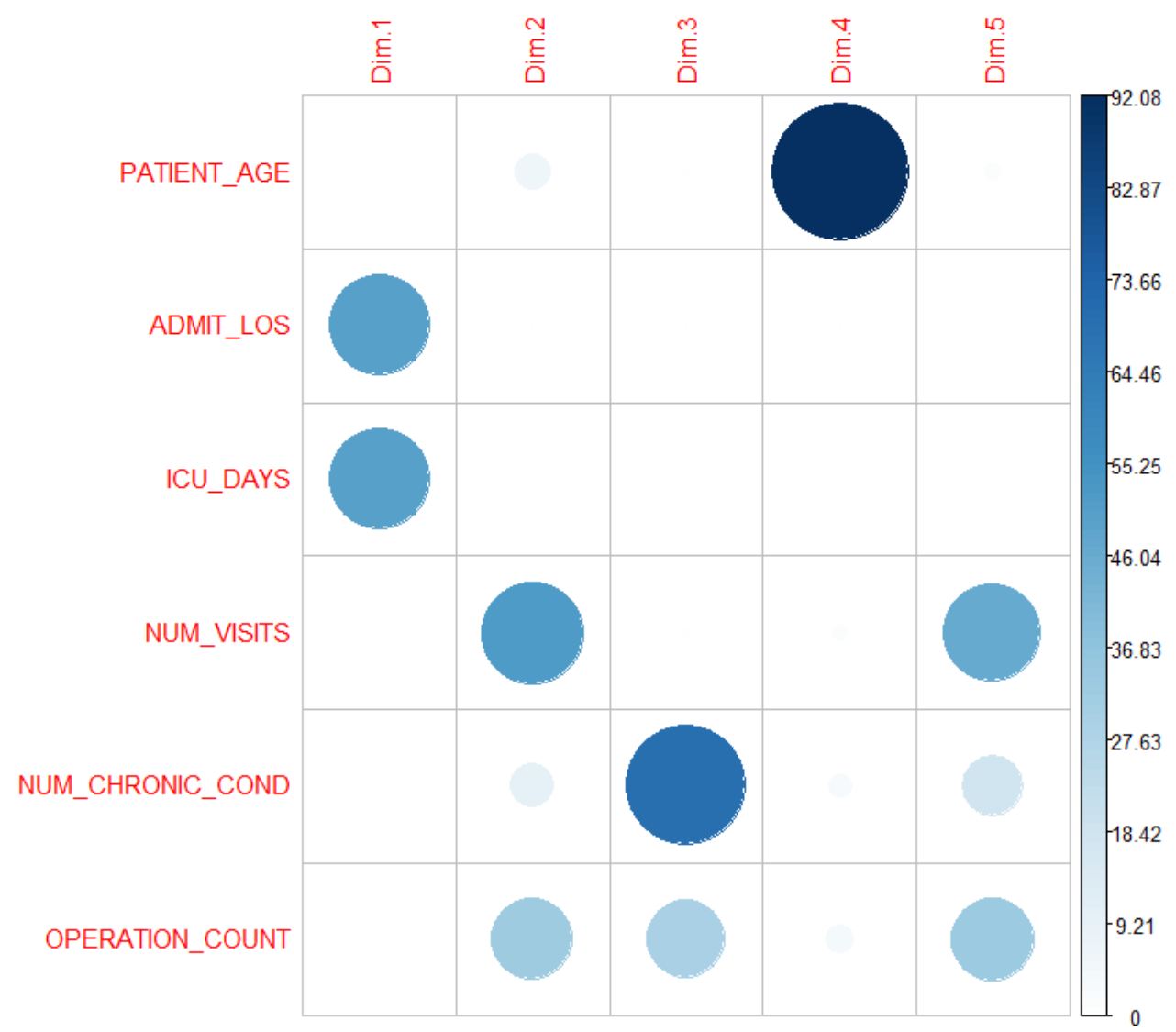

Figure 4. Principal components with respect to feature weights.

\section{Multivariate logistic regression}

The glm2 package was installed and subsequently called in R studio. Scripts were written to compute the logistic regression with readmit_number (our target) being the response variable $\mathrm{Y}$ and other regressor (predictor) variables X (e.g. gender and order_total_charges). The model was subsequently validated with the $30 \%$ validate dataset that was originally separated from the $90 \%$ partitioned datasets.

\section{Automated Variable Selection}

We also attempted to determine the drivers for readmission by using the automated variable selection (AVS) method. The AVS method was computed using the stepwise approach. 


\section{Issues in Information Systems}

Volume 22, Issue 4, pp. 334-345, 2021

Table 1. Combined results for all techniques with corresponding Area Under Curve (AUC) where applicable

\begin{tabular}{|c|c|c|}
\hline Model & Input & AUC (Target is 1) \\
\hline 1 & $\begin{array}{l}\text { Patient Age, Operations Count, Number of Visits } \\
\text { ICU Days, Num of Chronic Conditions, Admit Length of Stay }\end{array}$ & 0.5877 \\
\hline 2 & $\begin{array}{l}\text { Operations Count, ICU Days } \\
\text { Num of Chronic Conditions, Admit Length of Stay }\end{array}$ & 0.5880 \\
\hline 3 & $\begin{array}{l}\text { Operations Count } \\
\text { ICU Days } \\
\text { Num of Chronic Conditions }\end{array}$ & 0.5865 \\
\hline 4 & $\begin{array}{l}\text { Patient Age } \\
\text { Operations Count } \\
\text { ICU Days }\end{array}$ & 0.5836 \\
\hline 5 & $\begin{array}{l}\text { Operations Count } \\
\text { ICU Days } \\
\text { Admit Length of Stay }\end{array}$ & 0.5841 \\
\hline Auto & $\begin{array}{l}\text { Patient Age, Operations Count, Number of Visits } \\
\text { ICU Days, Num of Chronic Conditions, Admit Length of Stay }\end{array}$ & 0.5877 \\
\hline PCA & $\begin{array}{l}\text { Patient Age, Operations Count, Number of Visits } \\
\text { ICU Days, Num of Chronic Conditions, Admit Length of Stay }\end{array}$ & N/A \\
\hline
\end{tabular}

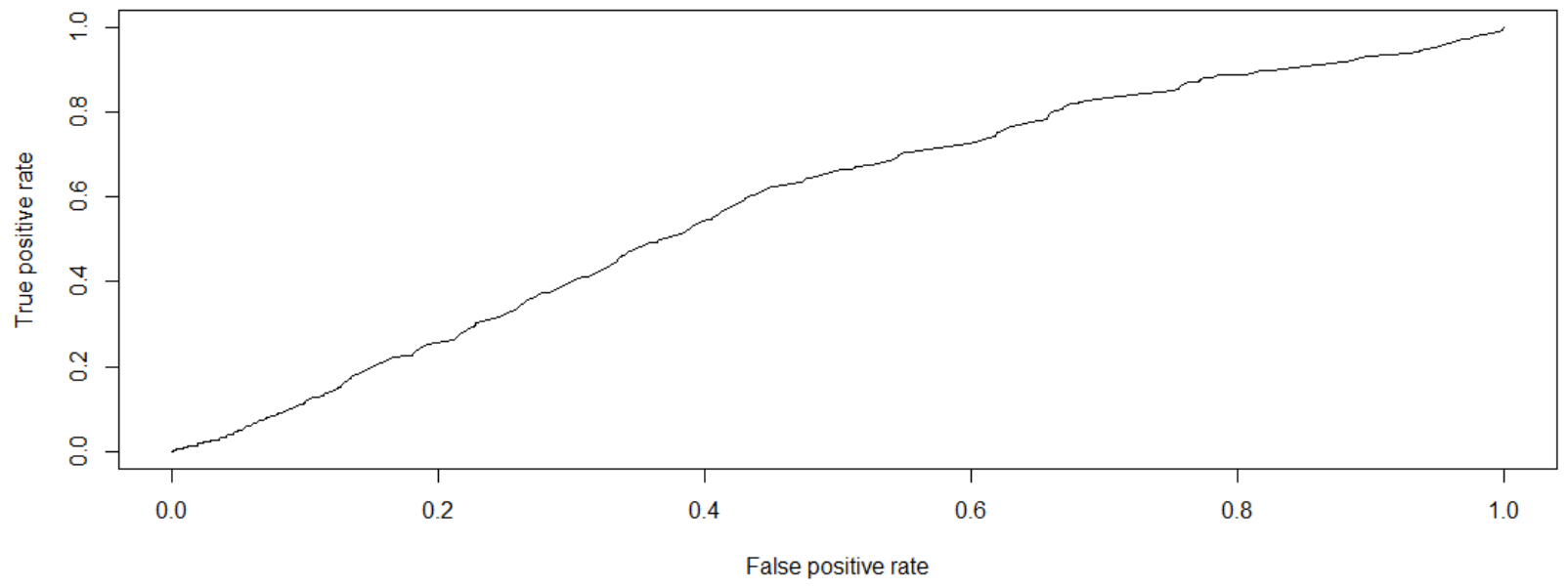

Figure 5. The True Positive and False Positive rates Area under curve (AUC) is good measure of model accuracy with maximum value 1.

\section{Results Interpretations and Discussion}

Table 1 above indicated very similar AUC values between the models. In fact, model 2 seems to be slightly better than the other models. However, with the previous results from the test of correlation and PCA, ICU_Days and length of Stay variables highly correlate. Having established that they both highlight similar information in the dataset (See Figure 2), we decided that it was not necessary to include both variables in the same model. There was not much differences between the models' respective AUCs, with the exception that model 2 appears to be slightly better, hence model 2 was selected as shown. In all respects, the PCA, Multivariate and Auto Regression models appear to agree on the most important variables to include in the models. The results showed that the training dataset was validated. Selected models indicate that variables, 


\section{Issues in Information Systems}

Volume 22, Issue 4, pp. 334-345, 2021

such as number of operations that the patient went through, time spent in the ICU, number chronic conditions, length of stay at the hospital, are the major drivers of the likelihood that a patient will be readmitted. Other lesser drivers include Patient's age, medical facility discharged to (rehab, hospice medical inpatient, hospital department (e.g. heart), specific hospital locations, states (i.e. Georgia and Florida). Hospital was not a predictor variable for determining probability of readmission event.

There is typically an interplay of factors that determine whether a patient recovers or returns for readmission. Basu, Avila \& Ricciardi (2016) described a conceptual framework for readmission which combines previous frameworks from Andersen-Newman (Andersen, 1995), Structure-Process framework (Aday et al., 1998), and Ashton and Wray (1996) to show a combination of patients and community features that may synergistically act to produce hospital readmission. These features include predisposing (gender, race, age), enabling (income, insurance, literacy), need (health status, prevalence of chronic illness), and environmental (resource supplies, primary care provider and population density, and geographic location). Other contributing factors include those associated with hospital stay etc. Several of the major and minor drivers identified can be controlled by the hospital through early intervention programs that should start through education of patients and caretakers before discharge. There should also be an on-going intervention program including follow-up to make sure that patients are following established care-plan and routine that may include exercise, medication use, complication avoidance, follow-up patient visit to the hospital, and nutrition among others. Why are these findings important?

The need for improved healthcare systems is a concerted effort that involves every stakeholder including insurance companies, policy makers and hospital management amongst others. In terms of quality assurance, patient outcome and cost reduction, hospital readmission has come under focus in the past several years and thus has ramifications for stakeholders. The patients suffer immensely from inconvenience to infection, injuries and even mortality. Modern day policies penalize health systems for quality issues including readmission within 30-days, and rising healthcare costs. For instance, Medicare uses a "all-cause" definition for readmission, that is, readmission is triggered when a patient returns to stay in the hospital within 30 days of an initial discharge (Boccuti \& Cassilas, 2015). The only exception to this, is when a patient returns within the 30-day window because of a pre-planned hospitalization, for instance, a scheduled coronary procedure. As a result of the potential penalties earlier described, hospital systems have an incentive to improve quality of care in order to minimize readmissions. Although one might argue against the practice of penalizing hospitals for readmissions because it seems to absolve other stakeholders including the patients of any responsibility especially because hospitals do not have a full control over the patient's care once discharged. However, experience shows that hospitals can have joint collaborative efforts with other stakeholders in terms of providing care plans with clear instructions for the patient on discharge; coordinating with post-acute care providers such as home care agencies; and by reducing to the barest minimum, the potential for complications during the initial hospital admission.

Several authors have observed that readmission rates started to decline for patients that had been hospitalized for heart conditions and pneumonia (Boccuti \& Cassilas, 2015). In fact, in order to keep systems working to decrease readmission, the goalpost is designed to keep moving. Boccuti \& Cassilas (2017) in their follow-up presentation on the subject, implied that, among penalized hospitals, the average fine increased by 13 percentage points from 2016 to 2017 when the average fine was increased to $0.74 \%$ reduction in Medicare inpatient payment. As a result of these ramifications, hospital systems have considerably large incentives to mine and utilize historical patients' data for predictive analytics and decision making with the objectives of using results for strategic planning and implementing resourceful health programs. 


\section{Issues in Information Systems}

Volume 22, Issue 4, pp. 334-345, 2021

Hospital systems have improved on data collection in the past decade. Hospital datasets are "gold mines" that can continue to assist in creating informed policies and making impactful decision for further lowering readmissions. This is especially important because readmission rates (apart from those established by the CMS or government) for each hospital should not be fixed but a "moving target." If there is a minimum requirement, there is a tendency for systems to flatten out once requirements are met. However, as better tools and analytical techniques are engaged, hidden patterns can be revealed and analytical modeling can impact decision making toward enacting and implementing policies that can keep healthy out-patients away from the hospital. This is a demonstration at the core of this research in using multiple techniques to investigate a typical readmission dataset.

\section{Conclusions}

Approximately $30 \%$ of the US population has multiple chronic conditions responsible for $70 \%$ of health care expenditures. Using the datasets from a hospital system in the South East of the United States, we found that readmission probability is mainly driven by several factors including operations count, number of days spent in the ICU, num of chronic conditions, and hospital length of stay. Results emphasize the importance of predictive analytics and multiple techniques to inform health policies, intervention planning, empower hospital management to help enhance care in a bid to keep minimizing hospital readmissions and care costs.

\section{References}

Aday, L. A., Begley C. E., Lairson D. R., and Slater C. H.. 1998. Evaluating the Healthcare System: Effectiveness, Efficiency, and Equity. Chicago, IL: Health Administration Press.

AHRQ. 2004. Agency for Healthcare Research and Quality: Healthcare Cost and Utilization Project (HCUP). Rockville, MD: AHRQ; [accessed on April 1, 2015]. Available at http://www.hcupus.ahrq.gov/db/state/siddbdocumentation.jsp

Ajani T., \& Habek G. 2019. A Machine Learning Approach to Optimizing Diabetes Healthcare Management Using SAS Analytic Suite. Journal of Information Systems Applied Research. Volume 12, No. 2 August 2019 ISSN: 1946-1836.

Andersen, R. M. 1995. "Revisiting the Behavioral Model and Access to Medical Care: Does it Matter?" Journal of Health and Social Behavior 36 (March): 1-10.

Ashton, C. M., and Wray N. P.. 1996. “A Conceptual Framework for the Study of Early Readmission as an Indicator of Quality of Care." Social Science and Medicine 43 (11): 1533-41.

Barnett, M. L., Hsu, J., \& McWilliams, J. M. (2015). Patient Characteristics and Differences in Hospital Readmission Rates. JAMA internal medicine, 175(11), 1803-1812. https://doi.org/10.1001/jamainternmed.2015.4660

Basu, J., Avila, R., \& Ricciardi, R. (2016). Hospital Readmission Rates in U.S. States: Are Readmissions Higher Where More Patients with Multiple Chronic Conditions Cluster?. Health services research, 51(3), 1135-1151. https://doi.org/10.1111/1475-6773.12401

Blecker, S., Paul, M., Taksler, G., Ogedegbe, G., Katz, S. (2013). Heart Failure-Associated Hospitalizations in the United States J Am Coll Cardiol. 2013 Mar, 61 (12) 1259-1267. 


\section{Issues in Information Systems}

Volume 22, Issue 4, pp. 334-345, 2021

Blecker, S., Herrin, J., Li, Li., Yu, H., Grady, J.N., Horwitz, L.I. (2019). Trends in Hospital Readmission of Medicare-Covered Patients With Heart Failure. Retrieved from: https://www.sciencedirect.com/science/article/pii/S0735109719300993\#sec1.1

Boccuti \& Cassilas, (2015). Aiming for Fewer Hospital U-turns: The Medicare Hospital Readmission Reduction Program. The Henry Keiser Family Foundation. January 2015: Issue Brief

Boccuti \& Cassilas, (2017). Aiming for Fewer Hospital U-turns: The Medicare Hospital Readmission Reduction Program. Retrieved from: https://www.kff.org/report-section/aiming-for-fewerhospital-u-turns-the-medicare-hospital-readmission-reduction-program-appendix/

Desai NR, Ross JS, Kwon JY, et al. (2016). Association between hospital penalty status under the Hospital Readmission Reduction Program and readmission rates for target and nontarget conditions. JAMA. 2016;316(24):2647-2656.

Goel, N., Iyengar, A., Kelly, J.J., Brown, C.R., Kurshan, F., Atluri, P., Acker, M.A. Chen, Z., Desai, N.D. (2019). Causes, Risk Factors, and Costs of 30-DayReadmissions After Mitral Valve Repair and Replacement. Retrieved from: https://www.annalsthoracicsurgery.org/article/S0003$\underline{4975(19) 31258-5 / \mathrm{pdf}}$

Herrin, J., St Andre, J., Kenward, K., Joshi, M. S., Audet, A. M., \& Hines, S. C. (2015). Community factors and hospital readmission rates. Health services research, 50(1), 20-39. https://doi.org/10.1111/1475-6773.12177

Joynt, K.E., Orav, E.J. Jha, A.K. (2011). Thirty-Day Readmission Rates for Medicare Beneficiaries by Race and Site of Care. Journal of the American Medical Association. 2011;305(7):675-81.

Krumholz, H.M., Merrill, A.R., Schone, E.M., Schreiner, G.C., Chen, J., Bradley, E.H., Wang, Y., Wang, Y., Lin, Z., Straube, B.M., Rapp, M.T., Normand, S.L., Drye, E.E. (2009). Patterns of Hospital Performance in Acute Myocardial Infarction and Heart Failure 30-Day Mortality and Readmission. Circulation: Cardiovascular Quality and Outcomes. 2009;2(5):407-13.

Ma, C., Bao, S., Dull, P., Wu, B., Yu, F. (2019). Hospital readmission in persons with dementia: A systematic review. Int J Geriatr Psychiatry. 2019; 34: 1170- 1184. https://doi.org/10.1002/gps.5140

Merkow, R. P., Ju, M. H., Chung, J. W., Hall, B. L., Cohen, M. E., Williams, M. V., Tsai, T. C., Ko, C. Y., \& Bilimoria, K.Y. (2015). Underlying reasons associated with hospital readmission following surgery in the United States. JAMA, 313(5), 483-495. https://doi.org/10.1001/jama.2014.18614

Shaheen, A. A., Nguyen, H. H., Congly, S. E., Kaplan, G. G., \& Swain, M. G. (2019). Nationwide estimates and risk factors of hospital readmission in patients with cirrhosis in the United States. Liver international : official journal of the International Association for the Study of the Liver, 39(5), 878-884. https://doi.org/10.1111/liv.14054

Sheingold, S. H., Zuckerman, R., \& Shartzer, A. (2016). Understanding Medicare Hospital Readmission Rates and Differing Penalties Between Safety-Net And Other Hospitals. Health affairs (Project Hope), 35(1), 124-131. https://doi.org/10.1377/hlthaff.2015.0534 


\section{Issues in Information Systems}

Volume 22, Issue 4, pp. 334-345, 2021

Tsugawa, Y., Jena, A.B., Figueroa, J.F., Orav, E.J., Blumenthal, D.M., Jha, A.K. (2017). Comparison of Hospital Mortality and Readmission Rates for Medicare Patients Treated by Male vs Female Physicians. JAMA Intern Med. 2017;177(2):206-213. doi:10.1001/jamainternmed.2016.7875 